\title{
Lithic Technology
}

William Andrefsky, Jr. (ed.)

Cambridge, Cambridge University Press, 2008, 340 pp. (hardback), $\$ 76.00$.

ISBN-13: 978052188827.

\section{Reviewed by JOHN D. RISSETTO}

Department of Anthropology. University of New Mexico, Albuquerque, NM 87131, USA; John413@unm.edu

Lithic Technology, edited by William Andrefsky, Jr., examines the use of various analytical measures derived from life history and technological organization theory to interpret retouched lithic tools originating from diverse cultural and temporal contexts. These measures, such as lithic reduction sequencing, chaine opératoire, tool curation, tool production effects, retouch measurements, and raw material selection and use, are illustrated by experimental and/or archaeological data presented in this volume. The authors use these methods to assess how lithic-based cultures organized their lithic technology and how these organizational processes in turn help reconstruct the "life histories" of discarded lithic tools. Throughout the volume, the contributors demonstrate how lithic life history and technological organization can provide a solid theoretical foundation for the interpretation of technologic, economic, and social behaviors of lithic-based cultures.

The various chapters cover a diverse geographic and chronological range of retouched lithic tool production cultures. They include the French Paleolithic, Near Eastern Neolithic, and the North American (USA and Canada) Paleoindian through Historic, as well as the additional research areas of Mongolia, Australia, and Italy. This range of geographic and temporal investigations demonstrates the broad application of lithic life histories and technological organization studies. The volume contains 14 chapters divided into four parts: I) introduction, background, review; II) production, reduction, and retouch; III) new perspectives on lithic raw material and technology; and, IV) evolutionary approaches to lithic technologies.

Part I consists of two chapters (Andrefsky; Shott and Nelson) that present detailed introductions and backgrounds into the context, definition, and application of lithic life history and technological organization theory as they relate to the analysis of retouched lithic tools. In the opening chapter, Andrefsky defines lithic tool life history as the study of lithic tools as they transform in shape, form, and function during their production to discard cycles. He goes on to define technological organization as "a strategy that deals with the way lithic technology (acquisition through discard) is embedded within the daily lives and adaptive choices and decisions of tool markers and users" (p.4). In Shott and Nelson's chapter, they provide a similar definition and interpretation of lithic technological organization, but use reduction thesis in concert with lithic life history study. They define the reduction thesis as "the understanding that retouched tools vary progressively from first use to discard by decrease in size and change in form depending on extent and pattern of the resharpening that they experience" (p. 27). The authors also reexamine the idea of tool curation. The authors move away from the classic Binfordian overall definition and use Shott's (1996: 267) more operational definition which states that tool curation is "the degree of use or utility extracted, expressed as a relationship between how much utility a tool starts with - its maximum utility - and how much that utility is realized before discard." This concept dovetails nicely with the reduction thesis and study of lithic life history. Shott and Nelson also provide a thorough interpretation and critical review of each of succeeding chapter.

Part II includes six chapters (Eren and Prendergast; Wilson and Andrefsky; Hiscock and Clarkson; Blades; Quinn et al.; Harper and Andrefsky), each of which provides a detailed example of how lithic reduction measures are used to examine lithic tool production, reduction, and retouch. Eren and Prendergast compare three measures of retouch intensity (index of reduction, index of invasiveness, and estimated reduction percentage) to identify which measures of tool mass loss work most effectively on unifacial stone scrapers tools from both an experimental and artifact assemblage. The artifact assemblage is part of the Perigoridan component from the La Colombiére rock shelter in France. Their analyses conclude that no single measure is more effective in measuring mass loss. This conclusion is based on the observation that each index measures a different aspect of retouch mass loss. Their research provides a step forward in mass loss measuring accuracy. Wilson and Andrefsky use a newly developed biface-specific retouch index to separate retouch that occurred during biface production from retouch that occurred during biface use and maintenance. The index examines and compares the form, location, and quantity of retouch on an experimental biface assemblage. They conclude that their measure is moderately successful and that their results provide new empirical evidence for differentiating production retouch from use retouch on biface artifacts. Hiscock and Clarkson demonstrate that flake retouching on Middle Paleolithic scrapers in France is a main determinant of a tool's overall form and size. In addition, the state to which the tool is ultimately reduced has direct implications for the tool's functional interpretation. They conclude that the degree of reduction is determined by various material- and knapper-based conditions. Blades uses Old and New World examples to identify differences between the gross reduction patterns of entire assemblages 
(reduction) and the reductions of individual tools (retouch intensity) within those assemblages. He uses flake-core ratios, the size of cores or blanks, the amount of cortical cover, and retouch tool characteristics to compare the stone acquisition and utilization patterns between assemblages. Variations between assemblages are explored to identify similarities in subsistence-settlement systems. Quinn et al. investigate the retouch intensity of Pre-Pottery Neolithic A el-Khiam points to determine their functional history. The authors use an analytical measure specifically designed for this research question to assess a point's retouch and use pattern in order to determine its use life history as a drill or perforator. Their results recommend that retouch measures be constructed around specific tool types and/or intra-assemblage research questions in order to most effectively reconstruct past cultural behavior. Harper and Andrefsky use various retouch measures to show that the life histories of Archaic period dart points in the American Southwest included their reuse by later Puebloan cultures as cutting tools. This research demonstrates that measures of retouch pattern can expose changes in tool functionality within different temporal and cultural contexts.

Part III contains three chapters (Andrefsky; MacDonald; Bradbury et al.) that offer new perspectives in how lithic life history and technological organization are affected by lithic raw material selection and utilization. Andrefsky uses obsidian distance-to-source data from pithouse occupants in Oregon and ethnographic threshold values to identify local from nonlocal scales of acquisition. These scales are then used to examine aspects of tool retouch, resharpening, and discard within the local/non-local circulation ranges of the tool makers. He equates the clear pattern between source distance and reduction intensity to be an effect of resource supply. MacDonald examines the influence of raw-material quality, abundance, and distribution by comparing chert use patterns between two sites in West Virginia. He uses an invasiveness index along with assemblage measures to examine whether tool design and curation rate are affected by stone availability and/or source distance. He concludes that stone tool function plays a significant role in lithic raw material selection. Bradbury et al. investigate, through experimental analysis, how different raw materials and hammer types influence the prediction of original flake mass. The authors' used a three-part measure of raw material quality to account for variations within and between raw material types. They conclude that in order to accurately determine original flake mass, raw material type and platform thickness must be taken into account.

Part IV consists of three chapters (Prentiss and Clarke; Clarkson; and Goodale et al.), which each offer an evolutionary approach to the analysis of lithic technological organization and retouched tools. Prentiss and Clarke use ecological and evolutionary approaches to identify and explain variations in the technological organization of retouched lithic tools within two contexts from the Pacific Northwest. They discuss how change in lithic tool manufacture and retouch patterns can occur during times of stable resource management strategies. They assert that because artifact stylistic and functional characteristics are directly associated with a human adaptive response to their social, economic or environmental surroundings, they are also subject to selection. Clarkson's study combines lithic tool retouch intensity with artifact recycling, raw material selection, and provisioning tactics to demonstrate how huntergatherer cultures in northern Australia changed land use patterns. He uses an excavated tool assemblage as a way to link changes in tool morphology to changes in social and environmental conditions, such as symbolic engagement and risk management. Goodale et al. link optimality theory to lithic reduction analyses in order to examine the degree of diversity in hypothetical core reduction strategies. These hypothetical models are designed to predict when humans would favor either more or less systematic core reduction techniques by incorporating three factors that influence diversity - raw material availability, raw material quality, and the ratio of tool producers to tool users. Their conclusions suggest that the diversity of core reduction techniques in diverse temporal and cultural assemblages is primarily a reflection of these three factors.

Part I of Lithic Technology presents a thorough explanation of the natural synthesis between technological organization theory and life history studies. This linkage provides a solid theoretical foundation from which to build analytical measures of retouched lithic tools. However, both chapters also acknowledge many of the limitations associated with using technological organization as a one-size-fits-all theoretical construct for deciphering past human behavioral intent and meaning through the myriad of knapping processes. While many of the chapters in Part II demonstrate the depth and breadth of curation and tool reduction analytical measures, a common takeaway is present from each conclusion. This takeaway stresses that the analytical measures designed to address these specific research questions presented in this volume may or may not be exactly applicable to other temporal or cultural contexts without some thoughtful tweaking. This tweaking requires that each researcher take into account his/her own research question(s), lithic reduction process, and cultural/temporal contexts when establishing their own technological organization-based analytical measures and procedures. While this may seem straightforward, its reiteration is warranted within this context of limited theoretical and methodological options. Another comment regarding Part II includes the use of refitting analysis as a means to test the precision of reduction measures used on archaeological assemblages. While refitting analysis is routinely used in experimental research to compare hypothetical to actual results, it would be a worthwhile endeavor to compare the mass, size, and form predictions derived from reduction analyses on archaeological assemblages to the actual mass, size and form of tested artifacts reconstructed through refitting. This refitting-based cross-check could help refine and strengthen the analytical and predictive results of future reduction indices. In Part III, the link between raw material and technological organization is clearly developed and presented in the associated chapters. While many of these chapters discuss the 
effects of lithic raw material on technological organization, curation, and reduction measures from a primarily biface tool perspective, it would interesting to compare this mode of analysis to different initial core reduction processes (e.g., blade core vs. bifacial core). Do they suggest similar technological organization processes and life histories with respect to the form and shape of the final retouched tool? This comparison would be appropriate for cultures who simultaneously utilized both biface and blade core reduction techniques, such as early Paleoindian and Upper Paleolithic cultures. In Part IV, the application of evolutionary and environmental theory seems to this reviewer to be a novel and welcome inclusion to linking lithic analysis to a theoretical construct. These three chapters offer solid examples of how this connection can exist and how it can be developed and strengthened through future research.

Lithic Technology succeeds in its goal of combining unique temporal and cultural examples to demonstrate a link between technological organization theory and the reconstruction of lithic retouched tool life histories. The chapters in this volume successfully combine the technical as- pects of lithic tool analysis with anthropological questions in order to show how lithic artifacts can help archaeologists better understand behavioral differences between the diverse range of lithic-based cultures. While this volume is not recommended as the sole text for a beginning level lithic survey class, unlike Andrefsky (2005), it should be required reading for any upper division undergraduate or graduate lithics class where its chapters can be discussed, debated and used as reference points for future research. Lithic Technology also is highly recommended for anyone interested in reading about diverse analytical measures for retouched lithic tools and theoretical arguments regarding lithic production trajectories currently debated by lithicists around the globe.

\section{REFERENCES}

Andrefsky, W. 2005. Lithics: Macroscopic Approaches to Analysis. 2nd edition. Cambridge University Press, Cambridge.

Shott, M. 1996. An Exegesis of the Curation Concept. Journal of Anthropological Research 52: 259-280. 